\title{
ROMIFIDINA, BUPIVACAÍNA, FENTANIL OU SUAS ASSOCIAÇÕES PELA VIA INTRATECAL EM CÃES SRD, PRÉ-MEDICADOS COM ACEPROMAZINA E DIAZEPAM
}

\section{INTRATHECAL ROUTE ROMIFIDINE, BUPIVACAINE, FENTANYL OR ASSOCIATIONS ADMINISTRATION FOR MONGREL DOGS PREMEDICATED WITH ACEPROMAZINE AND DIAZEPAN}

\author{
Anderson FARIAS \\ Orientadora: Professora Dra. Itaíra SUSKO \\ Departamento de Medicina Veterinária - UFPR
}

\begin{abstract}
RESUMO
O alívio da dor em animais após uma injúria acidental ou cirúrgica é uma das maiores preocupações do Médico Veterinário e a administração epidural de agentes analgésicos têm se demonstrado efetiva no manejo da dor somatossensorial e visceral. Baseados nestas colocações realizou-se o experimento, com o objetivo de avaliar fisiologicamente os parâmetros anestésicos promovidos pelo uso de diversas drogas pela via intratecal em cães. Os animais foram divididos em seis grupos experimentais aqui denominados tratamentos $(T)$ sendo cada grupo $(n=6)$ submetido a um protocolo: T1: romifidina $(60 \mu \mathrm{g} /$ $\mathrm{kg})$, T2: bupivacaína $(1,0 \mathrm{mg} / \mathrm{kg})$, T3: fentanil $(5,0 \mu \mathrm{g} / \mathrm{kg})$, T4: bupivacaína + fentanil $(0,5 \mathrm{mg} / \mathrm{kg}+2,5 \mu \mathrm{g} / \mathrm{kg})$. T5: bupivacaína + romifidina $(0,5 \mathrm{mg} / \mathrm{kg}+30 \mu \mathrm{g} / \mathrm{kg})$ e T6: romifidina + fentanil $(30 \mu \mathrm{g} / \mathrm{kg}+2,5 \mu \mathrm{g} / \mathrm{kg})$. Na MPA foi utilizada acepromazina $(0,1 \mathrm{mg} / \mathrm{kg})$ e diazepam $(1,0 \mathrm{mg} / \mathrm{kg})$ IV. A avaliação paramétrica constou da aferição da freqüência cardíaca ( $F C)$; freqüência respiratória (f); temperatura retal $(\mathrm{T})$; oximetria (OX); pressões arteriais sistólica (PAS); média (PAM) e diastólica (PAD). Estes parâmetros foram mensurados

imediatamente antes e 15 min após MPA e 5 min da aplicação intratecal (M1), quando procedeu-se a mensuração dos reflexos anal e interdigital e das qualidades de analgesia e miorrelaxamento, observando-se num intervalo de 15 min durante um período total de 110 min compreendidos entre o $\mathrm{M} 2$ ao $\mathrm{M} 8$. Como resultado verificou-se que a associação de acepromazina e diazepam consistiu num excelente protocolo de contenção química. A Romifidina tanto isolada quanto em associação produziu um potente efeito antinociceptivo, mediado ao nível espinhal, enquanto que sua redistribuição levou a um estado hipnótico com efeitos cardiorrespiratórios signiticativos. O efeito da bupivacaína caracterizou-se pela hipotermia e hipotensão, potente bloqueio espinhal, excelente estado de miorrelaxamento e analgesia de longa duração. O fentanil isolado produziu poucas alterações cardiorrespiratórias, com uma boa analgesia de curta duração e sem perda de tônus muscular. O tratamento 4 (bupivacaína + fentanil) destacou-se pelo excelente padrão anestésico com mínima interferência nos parâmetros físicos.
\end{abstract}

\begin{abstract}
Alleviating pain in animals after accidental or surgical injury is a major concern of veterinary care providers. Epidural administration of analgesic agents has proven effective in management of somatosensory and visceral pain. The aim of this study was investigate six anesthetic protocols administered in spinal medulla by intrathecal route using six adult animals per group. T1: romifidine $(60 \mu \mathrm{g} / \mathrm{kg})$, T2: bupivacaine $(1,0 \mathrm{mg} / \mathrm{kg})$, T3: fentanyl $(5,0 \mu \mathrm{g} / \mathrm{kg})$, T4: bubivacaine + fentanyl $(0,5 \mathrm{mg} / \mathrm{kg}$ $+2,5 \mu \mathrm{g} / \mathrm{kg})$, T5: bupivacaine + romifidine $(0,5 \mathrm{mg} / \mathrm{kg}+30 \mu \mathrm{g} /$ $\mathrm{kg})$ e T6: romifidine + fentanyl $(30 \mu \mathrm{g} / \mathrm{kg}+2,5 \mu \mathrm{g} / \mathrm{kg})$. These animals were premedicated with acepromazine $(0,1 \mathrm{mg} / \mathrm{kg})$ and diazepam $(1,0 \mathrm{mg} / \mathrm{kg})$ IV. Heart and respiratory rates, temperature, $\mathrm{O}_{2}$ saturation, systolic, mean and diastolic pressures, interdigital and anal reflexes, muscle relaxation and coetaneous sensibility

were measured during anesthesia with $15 \mathrm{~min}$ of the interval during $110 \mathrm{~min}$. The acepromazine and diazepam produced an excellent chemical restraint. The Romifidine produced isolated or in association, at spinal level, a potent antinociceptive effect, while your redistribution carried a hypnotic state with significative cardiorespiratory effects. Fact observed in treatment 5 (bupivacaine + romifidine) and treatment 6 (fentanyl + romifidine). The bupivacaine characterized by hypothermia and hipotension, with potent spinal blocked, and good muscle relaxation and longer analgesia duration. The fentanyl produced minimal cardiorespiratory alteration, with good analgesia of the short duration and no loss muscle tonus. The treatment 4 (bupivacaine + fentanyl) detached by excellent anesthetic pattern with minimal cardiorespiratory alteration.
\end{abstract}

